\title{
A NEW APPROACH TO IMAGE RECONSTRUCTION FROM PROJECTIONS USING A RECURRENT NEURAL NETWORK
}

\author{
ROBERT CIERNIAK
}

\author{
Technical University of Czestochowa \\ Department of Computer Engineering \\ Al. Armii Krajowej 36, 42-200, Częstochowa, Poland \\ e-mail: cierniak@kik.pcz.czest.pl
}

\begin{abstract}
A new neural network approach to image reconstruction from projections considering the parallel geometry of the scanner is presented. To solve this key problem in computed tomography, a special recurrent neural network is proposed. The reconstruction process is performed during the minimization of the energy function in this network. The performed computer simulations show that the neural network reconstruction algorithm designed to work in this way outperforms conventional methods in the obtained image quality.
\end{abstract}

Keywords: image reconstruction from projections, neural networks, recurrent net.

\section{Introduction}

Since Cormack's publication (Cormack, 1963), one of the key tasks in the computed tomography has been to integrate many new algorithms into studies and apply them to the problem of reconstructing an image from projections. The most important reconstruction methods are those using convolution and back-projection (Jain, 1989; Lewitt, 1983; Ramachandran and Lakshminarayanan, 1971), Fourier inversion or an algebraic reconstruction technique (ART) (Censor, 1983; Gordon and Bender, 1970; Kaczmarz, 1937). Considering the increasing number of soft computing algorithms applicable to various science disciplines, it is possible that in the foreseeable future these algorithms will occupy an important place in computed tomography. In the framework of soft computing, the most popular approach to image reconstruction from projections is based on neural networks - a very popular and important tool of artificial intelligence systems for solving image processing problems, e.g., as described in (Cierniak and Rutkowski, 2000). The idea of a neural network applied to image reconstruction from projections is presented in (Kerr and Barlett, 1995a; Kerr and Barlett, 1995b; Kerr and Barlett, 1995c; Knoll et al., 1999; Munllay et al., 1994). Unfortunately, the supervised learning of algorithms described in these papers cannot lead to good performance. The main issue is the inability to learn all the possible image samples. Other structures were studied in the papers (Srinivasan and Han, 1993; Wang and Wahl, 1997). These structures represent the so-called algebraic approach to image reconstruction from projections. The main disadvantage of the algebraic approach applied to this problem is the huge size of the variable matrix which is used during the reconstruction process. In that case the computational complexity of the reconstruction process is proportional to the square of the image size multiplied by the number of projections. It directly affects the number of connections between the neurons in the net.

In order to overcome the above drawbacks of the existing algorithms, in this paper a new approach to tomographic image reconstruction from projections is developed and investigated. The network studied in the paper resembles a Hopfield structure. Similar structures were proposed in (Cichocki et al., 1995; Ingman and Merlis, 1992; Luo and Unbehauen, 1998) to solve the 1D signal reconstruction problem. That idea will be adopted to the algorithm of image reconstruction from projections in 2D. In the literature on the subject, two approaches to tomographic reconstruction algorithms dominate: transform methods and algebraic reconstruction techniques. In our paper, the neural reconstruction algorithm is very close to the transformation methodology, in contrast to algebraic algorithms using the neural network proposed in (Srinivasan and Han, 1993; Wang and Wahl, 1997). The approach presented in this paper significantly decreases the 
complexity of the reconstruction problem. In our case the number of neurons in the network is proportional only to the square of the image size. This means that the size of the neural network is independent of the resolution of the projections performed earlier. As a result, we have a drastic decrease in the number of connections in the neural network. The reconstruction method presented herein, originally formulated by the author, can be directly applied to parallel beam tomography (see, e.g., (Cierniak, 2002; Cierniak, 2006)). The weights of the neural network arising in our reconstruction method will be originally determined, taking into account the interpolation function which was used earlier in the back-projection operation. The calculations of these weights will be carried out before the principal part of the reconstruction process is started. It will be shown that the weights are the same for all the neurons in the network.

\section{Neural algorithm of image reconstruction from projections}

Our reconstruction algorithm resembles the $\rho$-filtered layergram method (Lewitt, 1983). The main difference between these two methods is the realization of the filtering. In our case a recurrent neural network is implemented instead of the two-dimensional filtering of the blurred image obtained after the back-projection operation. The idea of the presented reconstruction method using a neural network is shown in Fig.1, where the parallel-beam geometry of collected projections is taken into consideration.
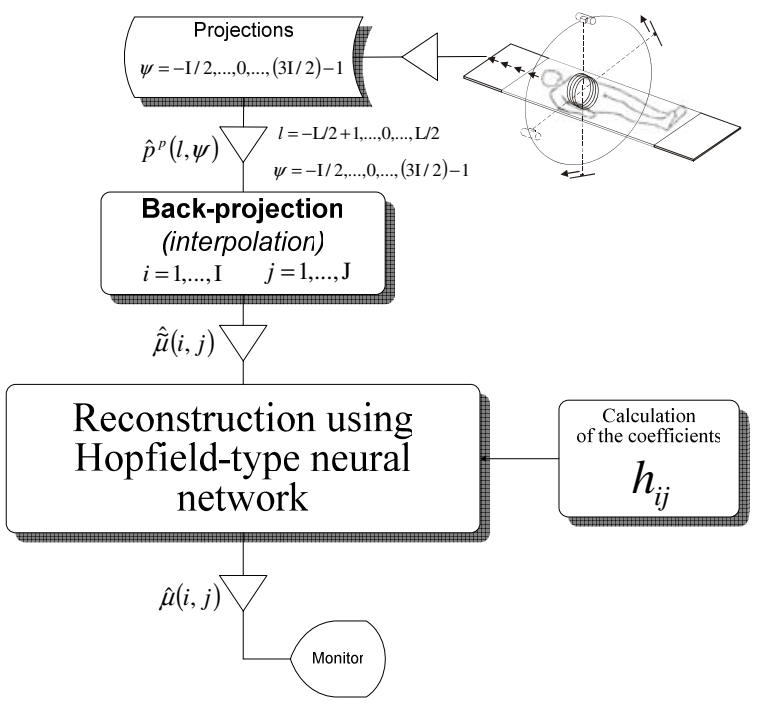

Fig. 1. Neural network image reconstruction algorithm using parallel beams.

2.1. Acquisition of projections. Only a limited number of parallel projections $p^{p}\left(s, \alpha^{p}\right)$ is chosen for further processing. Let $\hat{p}^{p}(l, \psi)$ denote discrete values of parallel projections taken at angles indexed by the variable $\psi$, where $\psi=-(I-1) / 2, \ldots, 0, \ldots,(3(I-1) / 2)-1$ and $2(I-1)$ is the number of projections. According to the concept of the discrete Radon transform (DRT) (Averbuch et al., 2001; Kingston and Svalbe, 2003), we choose only "grid friendly" angles of parallel projections, instead of an equiangular sample, considering the following condition for discrete values of the parameter $\alpha^{p}$ :

$$
\alpha_{\Psi}^{p}=\left\{\begin{array}{c}
\arctan \left(\frac{2 \psi}{I-1}\right)-\frac{\pi}{2} \\
\text { for } \Psi=-64, \ldots, 64, \\
\arctan \left(\frac{2(I-1-\Psi)}{I-1}\right)-\frac{\pi}{2} \\
\text { for } \Psi=65, \ldots, 191 .
\end{array}\right.
$$

The proposed distribution of the projection angles is approximately equiangular in the range of $\alpha^{p} \in$ $[-3 \pi / 4, \pi / 4)$, which is depicted clearly in Fig. 2.

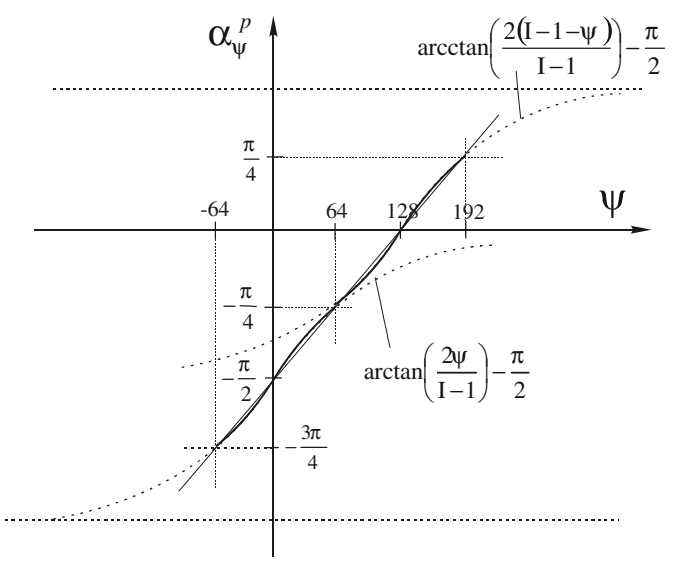

Fig. 2. The choice of parallel projection angles.

2.2. Operation of back-projection. In the next step we apply the back-projection operation (Jain, 1989; Lewitt, 1983). This can be performed in a continuous domain as follows:

$$
\tilde{\mu}(x, y)=\int_{0}^{\pi} p^{p}\left(s, \alpha^{p}\right) \mathrm{d} \alpha^{p}
$$

In a practical realization of the proposed reconstruction algorithm it is highly possible that for any given projection no ray passes through a given point $(i, j)$ of the image. To take this into account, we can apply interpolation. In this case a projection value mapped to a certain point of the reconstructed image is given by

$$
\bar{p}^{p}\left(s_{x y}, \alpha^{p}\right)=\int_{-\infty}^{\infty} p^{p}\left(s, \alpha^{p}\right) I(\dot{s}-s) \mathrm{d} s,
$$


where $p^{p}\left(s, \alpha^{p}\right)$ is expressed by the formula (1), $s_{x y}=$ $x \cos \alpha^{p}+y \sin \alpha^{p}$.

In the presented method we take into consideration discrete forms of images $\mu(x, y)$ and $\tilde{\mu}(x, y)$. This means that we will substitute the continuous functions of the images in (3) for their discrete equivalents $\hat{\mu}(i, j)$ and $\hat{\tilde{\mu}}(i, j)$, respectively, where $i=1,2, \ldots, I$ and $j=$ $1,2, \ldots, J . \quad I$ and $J$ are the numbers of pixels in horizontal and vertical directions, respectively. The discrete approximation of (3) is given by

$$
\begin{aligned}
& \hat{\bar{p}}^{p}\left(i \Delta_{s}^{p} \cos \psi \Delta_{\alpha}^{p}+j \Delta_{s}^{p} \sin \psi \Delta_{\alpha}^{p}, \psi\right) \cong \Delta_{s}^{p} \sum_{l}\left\{\hat{p}^{p}(l, \psi)\right. \\
&\left.\quad \times I\left(i \Delta_{s}^{p} \cos \psi \Delta_{\alpha}^{p}+j \Delta_{s}^{p} \sin \psi \Delta_{\alpha}^{p}-l \Delta_{s}^{p}\right)\right\},
\end{aligned}
$$

which is convenient from a computational point of view. In (4), $I(\Delta s)$ is an interpolation function, $\Delta s=$ $i \Delta_{s} \cos \alpha+j \Delta_{s} \sin \alpha-l \Delta_{s}$. If we use the linear interpolation function (Lewitt, 1983), then

$$
I_{L}(\Delta s)=\left\{\begin{array}{lll}
\frac{1}{\Delta_{s}}\left(1-\frac{\left|\Delta_{s}\right|}{\Delta_{s}}\right) & \text { if } & |\Delta s| \leq \Delta_{s} \\
0 & \text { if } & |\Delta s|>\Delta_{s} .
\end{array}\right.
$$

Equation (4) has only two terms and can be reformulated as (Kak and Slanley, 1988)

$$
\begin{aligned}
& \hat{\bar{p}}^{p}\left(s_{i j}, \psi\right) \\
& \cong \hat{p}^{p}\left(l^{\downarrow}, \psi\right)+\left(\frac{s_{i j}}{\Delta_{s}^{p}}-l^{\downarrow}\right)\left(\hat{p}^{p}\left(l^{\uparrow}, \psi\right)-\hat{p}^{p}\left(l^{\downarrow}, \psi\right)\right),
\end{aligned}
$$

where $s_{i j}=i \Delta_{i} \cos \psi \Delta_{\psi}^{p}+j \Delta_{j} \sin \psi \Delta_{\psi}^{p}, l^{\downarrow}$ is the highest integer value less than the value of the variable $s_{i j}, l^{\uparrow}=$ $l^{\downarrow}+1$.

The obtained image after the back-projection operation, taking into consideration the interpolation, can be expressed by the following equation:

$$
\tilde{\mu}(x, y)=\int_{0}^{\pi} \bar{p}^{p}\left(s_{x y}, \alpha^{p}\right) \mathrm{d} \alpha^{p} .
$$

In practice only a limited number of projections is performed at angles $\alpha_{\psi}^{p}$, where $\psi=$ $-(I-1) / 2, \ldots, 0, \ldots,(3(I-1) / 2)-1$ ( $I$ stands for the size of the processed image), and we can approximate integration over the angle $\alpha^{p}$ by a finite sum. In consequence, Eqn. (7) takes the following form:

$$
\hat{\tilde{\mu}}(i, j)=\sum_{\psi} \frac{1}{\Delta_{\alpha_{\psi}^{p}}^{p}} \hat{\bar{p}}^{p}\left(s_{i j}, \alpha_{\psi}^{p}\right),
$$

where $s_{i j}=i \Delta_{s}^{p} \cos \alpha_{\psi}^{p}+j \Delta_{s}^{p} \sin \alpha_{\psi}^{p}, \Delta_{\alpha_{\psi}^{p}}^{p}=\alpha_{\psi}^{p}-\alpha_{\psi-1}^{p}$.
2.3. Reconstruction using the recurrent neural network. The discrete image obtained after the backprojection operation $\hat{\tilde{\mu}}(i, j)$ includes information about the original image $\hat{\mu}(i, j)$ blurred by a geometrical term. Our task is to reconstruct the original image from the given form of $\hat{\tilde{\mu}}(i, j)$ using a recurrent neural network (Hopfield, 1982). Before we start the design process of this network, it is necessary to formulate a discrete reconstruction problem and, in particular, to calculate coefficients representing the geometrical term distorting the original image. In our approach we take into consideration the interpolation function used during the back-projection operation.

2.3.1. Discrete reconstruction problem. Owing to the relations (3) and (7), it is possible to define the image obtained after the back-projection operation, in the following way:

$$
\begin{aligned}
\tilde{\mu}(x, y)= & \int_{0}^{\pi}\left(\int _ { - \infty } ^ { \infty } \left(\int_{-\infty}^{\infty} \int_{-\infty}^{\infty}(\mu(\ddot{x}, \ddot{y})\right.\right. \\
& \left.\times \delta\left(\ddot{x} \cos \alpha^{p}+\ddot{y} \sin \alpha^{p}-\dot{s}\right) \mathrm{d} \ddot{x} \mathrm{~d} \ddot{y}\right) \\
& \times I(\dot{s}-s)) \mathrm{d} s) \mathrm{d} \alpha^{p},
\end{aligned}
$$

where $s=x \cos \alpha^{p}+y \sin \alpha^{p}$. The function $\tilde{\mu}(x, y)$ denotes a blurred image obtained after the operations of projection and back-projection. Interchanging the order of the integration and taking in account the independence of $\mu(\ddot{x}, \ddot{y})$ from variable $s$ allows us to rewrite (9) in the form

$$
\begin{aligned}
\tilde{\mu}(x, y)= & \int_{0}^{\pi}\left(\int_{-\infty}^{\infty} \int_{-\infty}^{\infty}(\mu(\ddot{x}, \ddot{y})\right. \\
& \times \int_{-\infty}^{\infty}\left(\delta\left(\ddot{x} \cos \alpha^{p}+\ddot{y} \sin \alpha^{p}-\dot{s}\right)\right. \\
& \times I(\dot{s}-s)) \mathrm{d} s) \mathrm{d} \ddot{x} \mathrm{~d} \ddot{y}) \mathrm{d} \alpha^{p} .
\end{aligned}
$$

It should be noted that to the blurred image $\tilde{\mu}(x, y)$ obtained after the back-projection operation only these places in the original image contribute for which

$$
\ddot{x} \cos \alpha^{p}+\ddot{y} \sin \alpha^{p}=\dot{s} .
$$

Assuming that the constraint (11) holds, we can transform (10) to

$$
\begin{aligned}
\tilde{\mu}(x, y)= & \int_{0}^{\pi}\left(\int_{-\infty}^{\infty} \int_{-\infty}^{\infty}(\mu(\ddot{x}, \ddot{y})\right. \\
& \times I\left(\ddot{x} \cos \alpha^{p}+\ddot{y} \sin \alpha^{p}-x \cos \alpha^{p}\right. \\
& \left.\left.\left.\quad-y \sin \alpha^{p}\right)\right) \mathrm{~d} \ddot{x} \mathrm{~d} \ddot{y}\right) \mathrm{d} \alpha^{p} .
\end{aligned}
$$


Again we interchange the order of integration taking into account the independence of the function $\mu(\ddot{x}, \ddot{y})$ from the variable $\alpha^{p}$. Thus

$$
\begin{array}{r}
\tilde{\mu}(x, y)=\int_{-\infty}^{\infty} \int_{-\infty}^{\infty} \mu(\ddot{x}, \ddot{y}) \\
\left(\int _ { 0 } ^ { \pi } I \left(\ddot{x} \cos \alpha^{p}+\ddot{y} \sin \alpha^{p}-x \cos \alpha^{p}\right.\right. \\
\left.\left.-y \sin \alpha^{p}\right) \mathrm{~d} \alpha^{p}\right) \mathrm{d} \ddot{x} \mathrm{~d} \ddot{y} .
\end{array}
$$

According to the discrete Radon transform methodology, in our approach only a limited number of projections are performed at "grid friendly" angles $\alpha_{\psi}^{p}$, where

$$
\psi=-(I-1) / 2, \ldots, 0, \ldots,(3(I-1) / 2)-1
$$

(Averbuch et al., 2001; Kingston and Svalbe, 2003). We can approximate the integration over the angle $\alpha^{p}$ by a finite sum. Additionally, we approximate the 2-D convolution using two finite sums of ranges from 1 to $I$ and from 1 to $J$. In this way, we reformulate (13) as follows:

$$
\hat{\tilde{\mu}}(i, j) \cong \sum_{\ddot{i}} \sum_{\ddot{j}} \hat{\mu}(\ddot{i}, \ddot{j}) h_{i j i \ddot{j}},
$$

where

$$
\begin{aligned}
h_{i j \dddot{I} j} \cong\left(\Delta_{s}\right)^{2} \sum_{\Psi} & \Delta_{\Psi}^{p} \hat{I}\left(\ddot{I} \Delta_{s} \cos \alpha_{\psi}^{p}+\ddot{j} \Delta_{s} \sin \alpha_{\Psi}^{p}\right. \\
& \left.-I \Delta_{s} \cos \alpha_{\Psi}^{p}-j \Delta_{s} \sin \alpha_{\Psi}^{p}\right) .
\end{aligned}
$$

Since the interpolation function $\hat{I}(\Delta s)$ is even, we can write

$h_{i j i j}=h_{i j i j}$

$=\left(\Delta_{s}\right)^{2} \sum_{\Psi} \alpha_{\Psi}^{p} \hat{I}\left(|i-\ddot{i}| \Delta_{s} \cos \alpha_{\Psi}^{p}+|j-\ddot{j}| \Delta_{s} \sin \alpha_{\Psi}^{p}\right)$.

Therefore, we are able to formulate a very convenient relation between the original image and that obtained after the back-projection operation in the form of

$$
\hat{\tilde{\mu}}(i, j) \cong \sum_{\ddot{i}} \sum_{\ddot{j}} \hat{\mu}(\ddot{i}, \ddot{j}) h_{\Delta i, \Delta j},
$$

where

$$
\begin{aligned}
& h_{\Delta i, \Delta j} \\
& \quad=\left(\Delta_{s}\right)^{2} \sum_{\Psi} \Delta_{\Psi}^{p} \hat{I}\left(\Delta i \Delta_{s} \cos \alpha_{\psi}^{p}+\Delta j \Delta_{s} \sin \alpha_{\Psi}^{p}\right) .
\end{aligned}
$$

As one can see from (17), the original image in a given cross-section of the object, obtained in the way described above, is equal to the amalgamation of this image and the geometrical distortion element expressed by
(18). Owing to the form of (17), the number of coefficients $h_{\Delta i, \Delta j}$ is reduced and, owing to (18), the values of these coefficients are easily calculated. The coefficients $h_{\Delta i, \Delta j}$ are used to determine the weights in the recurrent neural network.

2.3.2. Design of the recurrent neural network. The recurrent neural network structure presented in Fig. 5 was proposed for the first time in (Cichocki et al., 1995; Ingman and Merlis, 1992; Luo and Unbehauen, 1998). The network performs image reconstruction from projection by the deconvolution of the relation (17). This can be formulated as the following optimization problem:

$$
\min _{\mathbf{M}}\left(w \sum_{i=1}^{\mathrm{I}} \sum_{j=1}^{\mathrm{J}} f\left(e_{i j}(M)\right)\right)
$$

where $M=[\hat{\mu}(i, j)]$ is the matrix with elements from the original image of a given object, $\tilde{M}=[\hat{\tilde{\mu}}(i, j)]$ constitutes the matrix with elements from the distorted image of a given object, $\mathbf{H}=\left[h_{i j}\right]$ signifies the matrix of the reconstruction coefficients, $w$ stands for a suitable large positive coefficient, $f(\cdot)$ means a penalty function, and

$$
e_{i j}(\mathbf{M})=\sum_{\ddot{i}=1}^{I} \sum_{\ddot{j}=1}^{J} h_{\Delta i, \Delta j} \hat{\mu}(\ddot{i}, \ddot{j})-\hat{\tilde{\mu}}(i, j) .
$$

If the value of the coefficient $v$ tends to infinity or is suitably large, then the solution to the optimization problem (19) tends to the optimal one. Our research has shown that the following penalty function yields the best result:

$$
f\left(e_{i j}\right)=\lambda \ln \cosh \left(\frac{e_{i j}}{\lambda}\right), \quad \lambda>0 .
$$

Observe that the derivative of the function (21) is easily calculated and takes the form of

$$
\begin{aligned}
f^{\prime}\left(e_{i j}\right) & =\frac{\partial f\left(e_{i j}\right)}{\partial e_{i j}}=\tanh \left(\frac{e_{i j}}{\lambda}\right) \\
& =\frac{1-\exp \left(-2 e_{i j} / \lambda\right)}{1+\exp \left(-2 e_{i j} / \lambda\right)},
\end{aligned}
$$

where $\lambda$ is a slope coefficient.

Now we will formulate the energy function which will be minimized by a constructed neural network. Simultaneously, we will realize the deconvolution task (cf. (17)). The energy function is given by

$$
E^{t}=v \sum_{i=1}^{\mathrm{I}} \sum_{j=1}^{\mathrm{J}} f\left(e_{i j}\left(\mathbf{M}^{t}\right)\right)
$$

In order to find the minimum of (23), we determine the 
derivative

$$
\begin{aligned}
\frac{\mathrm{d} E^{t}}{\mathrm{~d} t} & =w \sum_{i=1}^{I} \sum_{j=1}^{J} \sum_{\ddot{i}=1}^{I} \sum_{\ddot{j}=1}^{J} \\
& =\frac{\mathrm{d} f\left(e_{i j}\left(\mathbf{M}^{t}\right)\right)}{\mathrm{d} e_{i j}\left(\mathbf{M}^{t}\right)} \frac{\mathrm{d} e_{i j}\left(\mathbf{M}^{t}\right)}{\mathrm{d} \hat{\mu}^{t}(\ddot{i}, \ddot{j})} \frac{\mathrm{d} \hat{\mu}^{t}(\ddot{i}, \ddot{j})}{\mathrm{d} t}
\end{aligned}
$$

or

$$
\begin{aligned}
\frac{\mathrm{d} E^{t}}{\mathrm{~d} t}= & -\sum_{\ddot{i}=1}^{I} \sum_{\ddot{j}=1}^{J} \frac{\mathrm{d} \hat{\mu}^{t}(\ddot{i}, \ddot{j})}{\mathrm{d} t} \\
& \left(-w \sum_{k=1}^{I} \sum_{l=1}^{J} \frac{\mathrm{d} f\left(e_{i j}\left(\mathbf{M}^{t}\right)\right)}{\mathrm{de} e_{i j}\left(\mathbf{M}^{t}\right)} \frac{\mathrm{d} e_{i j}\left(\mathbf{M}^{t}\right)}{\mathrm{d} \hat{\mu}^{t}(\ddot{i}, \ddot{j})}\right)
\end{aligned}
$$

where the index $t$ means the dependence of the variable denoted by this symbol during the reconstruction process. If we let

$$
\begin{aligned}
\frac{\mathrm{d} \hat{\mu}^{t}(\ddot{i}, \ddot{j})}{\mathrm{d} t} & =-w \sum_{i=1}^{I} \sum_{j=1}^{J} \frac{\mathrm{d} f\left(e_{i j}\left(\mathbf{M}^{t}\right)\right)}{\mathrm{d} e_{i j}\left(\mathbf{M}^{t}\right)} \frac{\mathrm{d} e_{i j}\left(\mathbf{M}^{t}\right)}{\mathrm{d} \hat{\mu}^{t}(\ddot{i}, \ddot{j})} \\
& =-w \sum_{i=1}^{I} \sum_{j=1}^{J} f^{\prime}\left(e_{i j}\left(\mathbf{M}^{t}\right)\right) h_{\Delta i, \Delta j},
\end{aligned}
$$

then (25) takes the form of

$$
\frac{\mathrm{d} E^{t}}{\mathrm{~d} t}=-\sum_{\ddot{i}=1}^{\mathrm{I}} \sum_{\ddot{j}=1}^{\mathrm{J}}\left(\frac{\mathrm{d} \hat{\mu}^{t}(\ddot{i}, \ddot{j})}{\mathrm{d} t}\right)^{2} .
$$

One can see that the right-hand side of (27) is always nonpositive, i.e., $\mathrm{d} E^{t} / \mathrm{d} t \leq 0$. Therefore, if $\mathrm{d} E^{t} / \mathrm{d} t=0$, then this means that $\mathrm{d} \hat{\mu}^{t}(i, j) / \mathrm{d} t=0$ and the minimum of $E$ is obtained. At this moment, calculations for each pixel $(i, j)$ are completed.

The neural network performing the minimization task consists of two layers, with the same topology of neurons. The structure is shown in Fig. 3. All of the symbols which appear in the neural network structure are listed in order from the input to the output of the network.

First layer:

- weights of the connections:

$$
w_{i j i \dddot{j} j}^{(1)}=h_{i-\ddot{i}, j-\ddot{j}},
$$

- weighting sums:

$$
s_{i j}^{(1)}=e_{i j}^{t}(\mathbf{F})^{t}=\sum_{\ddot{i}} \sum_{\ddot{j}} w_{i j i j}^{(1)} \hat{\mu}^{t}(\ddot{i}, \ddot{j})-\hat{\tilde{\mu}}(i, j),
$$

- neurons outputs:

$$
y_{i j}^{(1)}=f^{(a 1)}\left(s_{i j}^{(1)}\right)=\frac{\mathrm{d} f\left(s_{i j}^{(1)}\right)}{\mathrm{d} s_{i j}^{(1)}}=\tanh \left(\frac{s_{i j}^{(1)}}{\lambda}\right) .
$$

Second layer:

- weights of the connections:

$$
w_{\ddot{i}, \ddot{j}, i, j}^{(2)}=-w \frac{\delta e_{i}^{t} j}{\delta \hat{\mu}(\ddot{i}, \ddot{j})}=-w \cdot h_{\ddot{i}-i, \ddot{j}-j},
$$

- weighting sums:

$$
\begin{aligned}
s_{i j}^{(2)} & =\sum_{i} \sum_{j} w_{i j}^{(2)} f^{(a 1)}\left(s_{i j}^{(1)}\right) \\
& =\sum_{i} \sum_{j} w_{\ddot{i}, \ddot{j}, i, j}^{(2)} y_{i j}^{(1)}, \\
s_{\dddot{i} j}^{(2)} & =\frac{\mathrm{d} \hat{\mu}^{t}(\ddot{i}, \ddot{j})}{\mathrm{d} t},
\end{aligned}
$$

- neurons outputs:

$$
\hat{\mu}^{t}(\ddot{i}, \ddot{j})=f^{(a 2)}\left(s_{i j}^{(2)}\right)=\int_{0}^{t} s_{i j}^{(2)} \mathrm{d} t .
$$

\section{Experimental results}

The size of the processed image was fixed at $I \times J=$ $129 \times 129$ pixels, which determines the number of neurons in each layer of the net. Before the reconstruction process using a recurrent neural network is started, it is necessary to calculate coefficients $h_{i j}$ using (27). In the case of using linear interpolation, the values of these coefficients for $j=0$ are presented in Fig. 4.

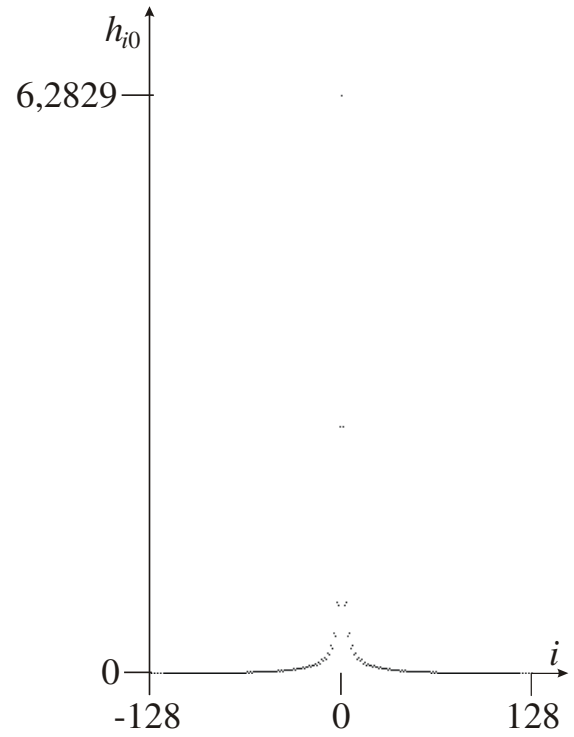

Fig. 4. Values of the coefficients $h_{i 0}$.

Owing to the lack of physical projection data from the tomograph, it is necessary to construct a mathematical model of the projected object, the so-called phantom. This 


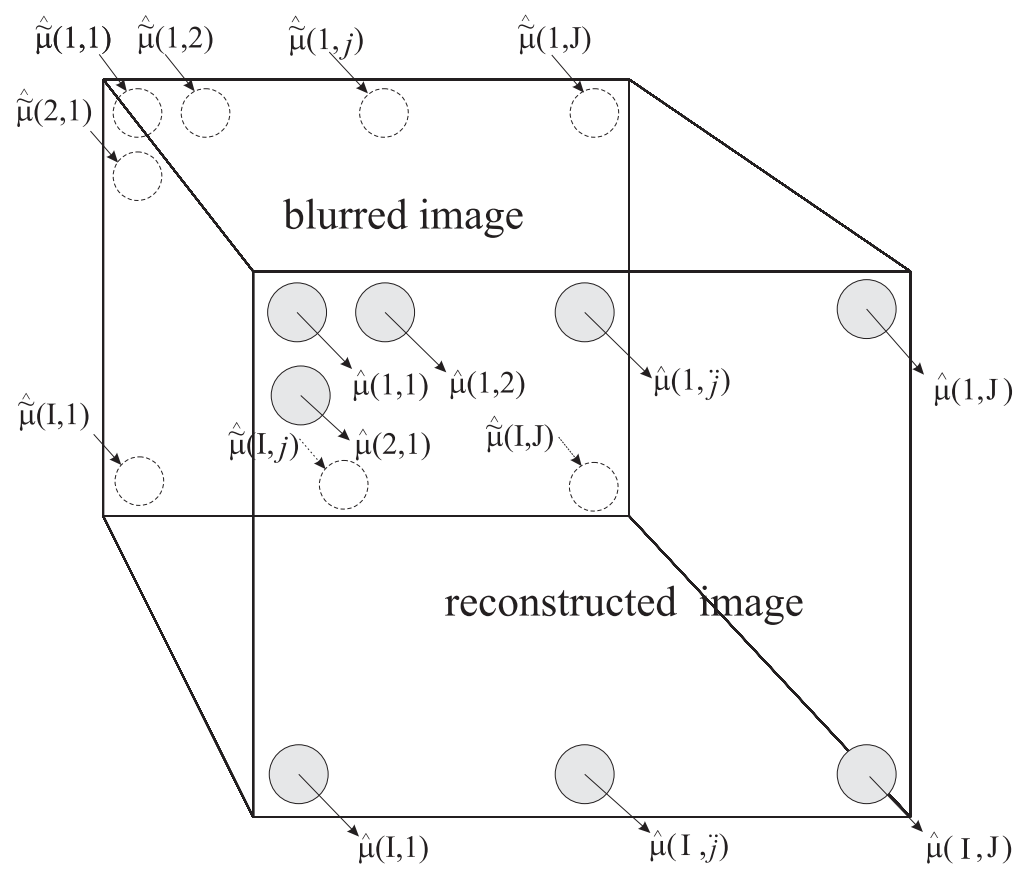

(a)

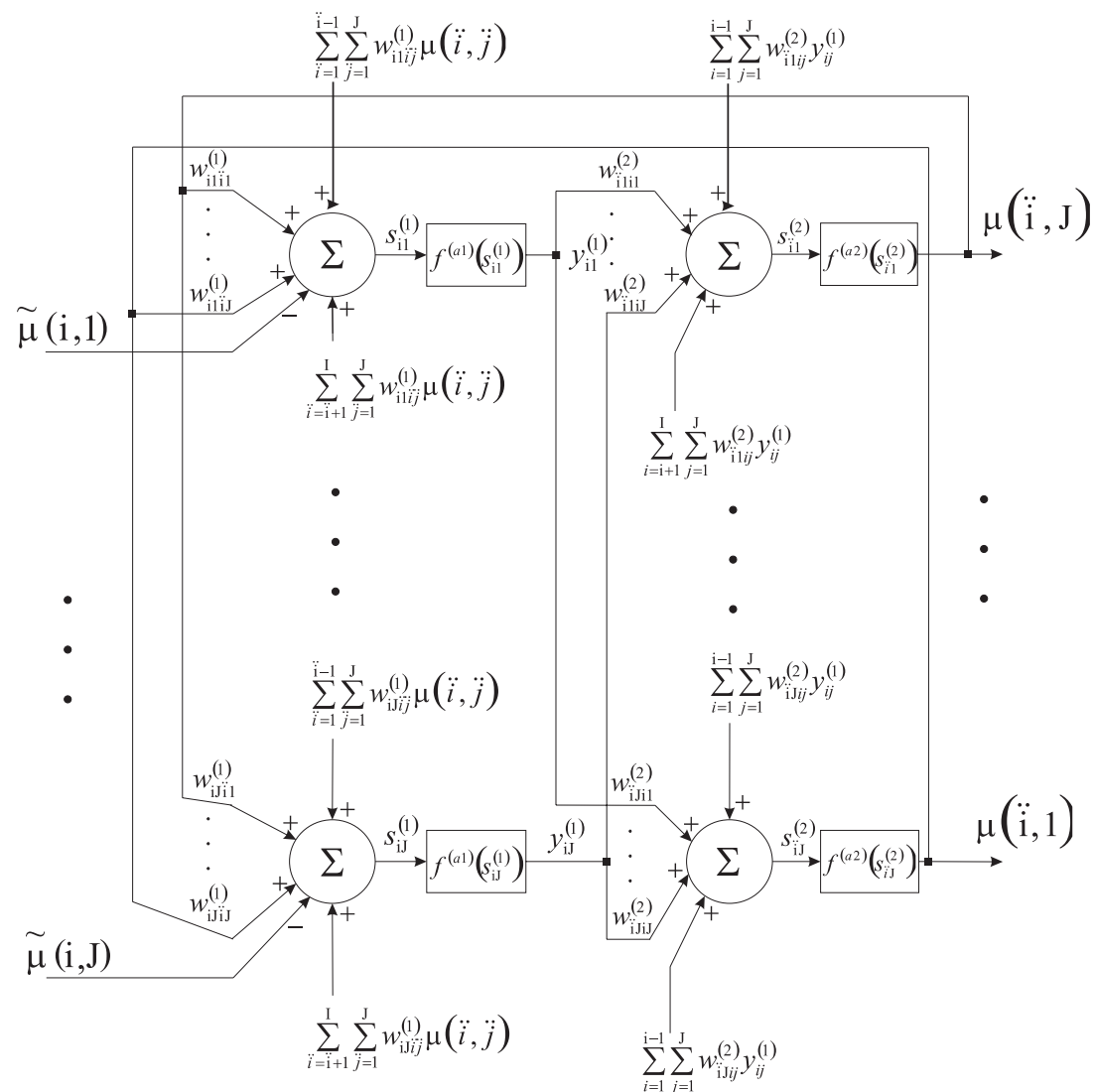

(b)

Fig. 3. Structure of the recurrent neural network in question: (a) topology of neurons in the net, (b) scheme of connections in the net. 


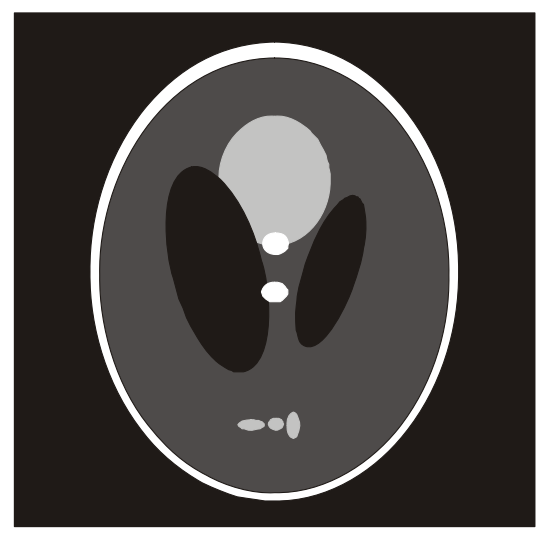

Fig. 5. A view obtained from the mathematical model of the cross-section of a skull.

example was proposed in the books (Jaene, 1991; Jain, 1989), see Fig. 5.

To estimate a particular projection obtained at any angle, we have to calculate the projections for each element of the model separately and then add them to each point of the screen. This means that calculating the projection values for an element of the model requires calculating the length of the path which a given ray passes across an element of the model at a given angle and then multiplying this length by the attenuation coefficient of the element. After calculating the projection values for each element of the mathematical model at certain points on the screen (where radiation detectors are located), these projections are summed. During the simulations we used 183 measure points (detectors) on the screen and the projections were obtained at 128 rotation angles with a total rotation angle of $180^{\circ}$.

The proposed form of interpolation presented in (14) could be applied to obtain the values of projections assigned to each pixel of the image.

After collecting the projections at every point of the image (operation of back-projection) we obtain the distribution of the $x$-ray attenuation coefficient in a given cross-section of the investigated object. A mathematical model with distortion is shown, after appropriate scaling, in Fig. 6.

In this way the obtained image was subjected to the process of reconstruction using a neural network whose structure was explained in the previous section. In an experimental way the values of the weight $w$ were selected at $w=6.2 \times 10^{10}$, with the slope at the level of $\lambda=10^{10}$. These two parameters determine the speed of the reconstruction process. A greater value of parameter $w$ at the same value of parameter $\lambda$ leads to instabilities during the numerical simulations. The progress in the reconstruction process is presented in Fig. 7, where the left-hand side figures represent views of the reconstructed images

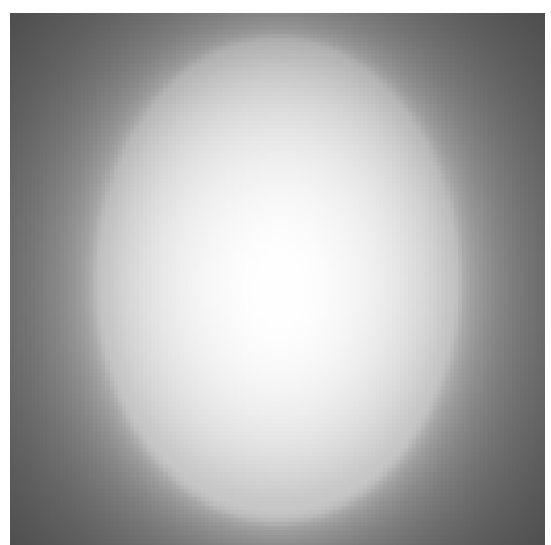

Fig. 6. A distorted image of the mathematical model obtained after projections.

for the parameters of the window (Kak and Slanley, 1988) $C=1.0, W=0.11$, and the right-hand side figures show central vertical cross-sections of the reconstructed images.

One can see above that the result after about 9,000 iterations of the reconstruction process is stabilized at a satisfactory level. Therefore, at this point the image is reconstructed and the process can be stopped.

The quality of the reconstructed image was evaluated in this case by error measures defined as follows:

$$
\begin{gathered}
M S E=\frac{1}{I J} \sum_{i=1}^{I} \sum_{j=1}^{J}[\mu(i, j)-\hat{\mu}(i, j)]^{2}, \\
S N R=10 \log _{10}\left(\frac{\sum_{i=1}^{I} \sum_{j=1}^{J}[\mu(i, j)]^{2}}{\sum_{i=1}^{I} \sum_{j=1}^{J}[\mu(i, j)-\hat{\mu}(i, j)]^{2}}\right),
\end{gathered}
$$

where $\mu(i, j)$ is the original image of the Shepp-Logan mathematical phantom.

Consider the case where projections $p(s, \alpha)$ are corrupted by additive Gaussian noise. For the simulations we can generate noisy projections $p^{N}(s, \alpha)$ using

$$
P^{N}(s, \alpha)=\left(1+N\left(p_{0}, \sigma^{2}\right)\right) p(s, \alpha),
$$

where $N\left(p_{0}, \sigma^{2}\right)$ is a Gaussian probability distribution with mean $p_{0}$ and variance $\sigma^{2}$ (in our simulations, we set $p_{0}=0$ and $\sigma^{2}=0.0025$ ).

At the beginning of simulations, we determined the value of the weight $w$. The results of experiments for different values of $w$ are depicted in Fig. 8.

Simulations show that if the weight $w$ is higher, then the reconstruction process is quicker. For $w=6.2 \times 10^{10}$ this reconstruction process becomes unstable. Figures 9 (projections without noise) and 10 (noisy projections) 
present a comparison of the simulation results (the lefthand figures represent views of the reconstructed images for window parameters (Kak and Slanley, 1988) $C=$ $1.0, W=0.11$ and the right-hand side figures show central vertical cross-sections of the reconstructed images).

For comparison, the reconstructed images obtained using the convolution back-projection algorithm (SheppLogan convolution kernel) under the same noisy projections are shown in Figs. 9(b) and 10(b). The results of this standard approach and results achieved by neural network methods described in this paper are presented in Table 1.

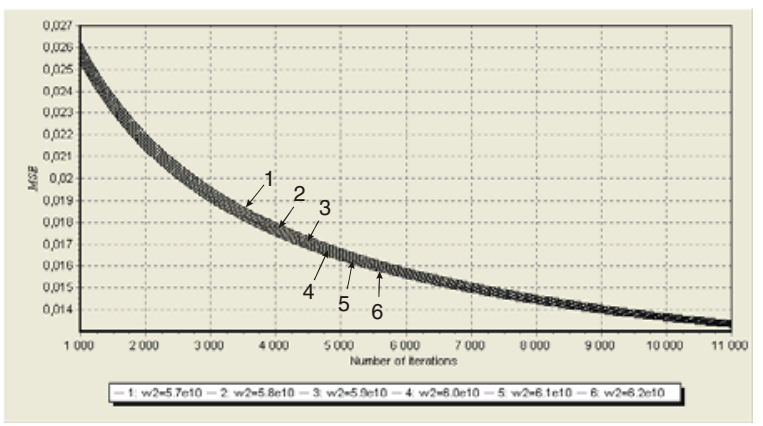

Fig. 8. Progress of the reconstruction process depending on the value of the weight $w$.

A very important factor of image reconstruction from the projection method is its algorithmic complexity. In the case of a fully parallel implementation of the neural approach presented in this paper, the computational time depends only on the used frequency and on the numbers of performed iterations. For comparison, in the case of the standard convolution/back-projection method the computational time depends on $\Psi \cdot 2 \cdot I^{2}$ additions and multiplications, where $I$ is the dimension of the processed image and $\Psi$ means the number of projections. For example, in the experiments described above, the computational time of our method is proportional to 30000 iterations and for the referential convolution/back-projection algorithm it is proportional to 4260096. The same back-projection operation is performed in both compared methods and is not taken into consideration for the comparison.

\section{Conclusions}

The performed simulations demonstrated the convergence of the image reconstruction algorithm based on the proposed recurrent neural network. The image of the crosssection of the investigated mathematical model obtained after a sufficient number of iterations is reconstructed with a high objective fidelity.

In comparison with the previous neural approaches to algebraic reconstruction techniques presented in (Srinivasan and Han, 1993; Wang and Wahl, 1997), one can observe a dramatic decrease in the number of neurons in the Hopfield-type network described in this work. More precisely, the size of each layer in our network was equal to $129 \times 129$ neurons. In previous approaches (Srinivasan and Han, 1993; Wang and Wahl, 1997), under the same conditions of the reconstructed image and projections, the number of neurons was equal to $129 \times 129 \times 183 \times 720$. That number was further reduced in (Srinivasan and Han, 1993) by a factor of $10^{2}$, but it is a very small profit compared with the results of our paper.

The algorithm described in this paper outperforms standard reconstruction methods in the sense of the mean square error. It should be noted that the simulations were performed using a sequential realization of the image reconstruction algorithm, as opposed to the natural parallel calculations in neural networks.

Therefore, a hardware realization of our neural network structure which keeps the parallel signal processing, e.g., by effective implementation using VLSI could give incomparably better results than the other methods of image reconstruction from projections concerning the time and quality of reconstruction.

Having a solution to the problem of image reconstruction from projections for parallel beams, one can extend our results to other geometries of projections: fanbeams and cone-beams, in particularly incorporated in spiral tomography. That means a possibility to implement a recurrent neural network in new designs of tomograph devices.

\section{References}

Averbuch A., Coifman R.R., Donoho D.L., Israeli M. and Waldén J. (2001). A notion of Radon transform for data in a Cartesian grid, which is rapidly computable, algebraically exact, geometrically faithful and invertible, Technical report TR No. 2001-11, Department of Statistics, Stanford University, USA.

Censor Y. (1983). Finite series-expansion reconstruction methods, Proceeding of the IEEE 71(3): 409-419.

Cichocki A., Unbehauen R., Lendl M. and Weinzierl K. (1995). Neural networks for linear inverse problems with incomplete data especially in application to signal and image reconstruction, Neurocomputing 8: 7-41.

Cierniak R. and Rutkowski L. (2000). On image compression by competitive neural networks and optimal linear predictors, Signal Processing: Image Communication 15(6): 559-565.

Cierniak R. (2002). Image reconstruction from projection using unsupervised neural network, Proceedings of the Joint 1st International Conference on Soft Computing and Intelligent Systems and 3-rd International Symposium on Advanced Intelligent Systems, Tsukuba, Japan.

Cierniak R. (2006). A novel approach to image reconstruction from projections using Hopfield-type neural network, in Rutkowski L., Tadeusiewicz R., Zadeh L. A., Żurada J. (Eds.), Proceedings of the 8-th International Conference 

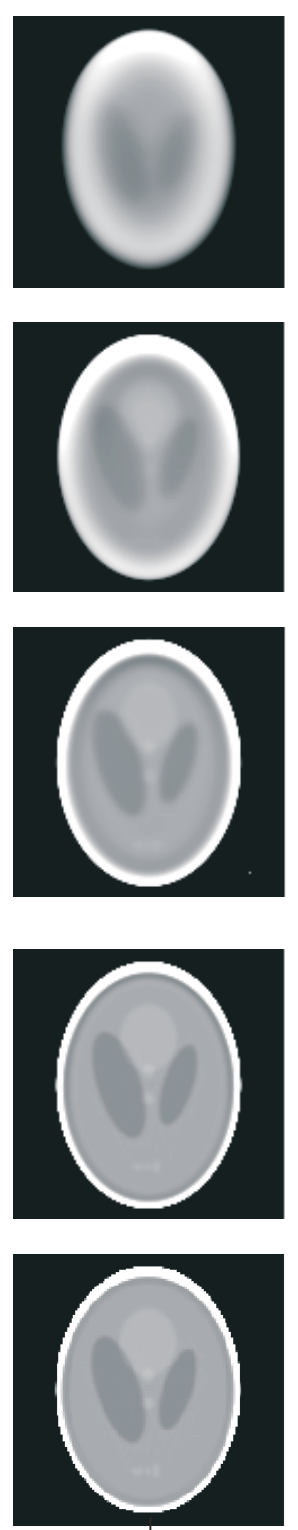

$\mathrm{A}$

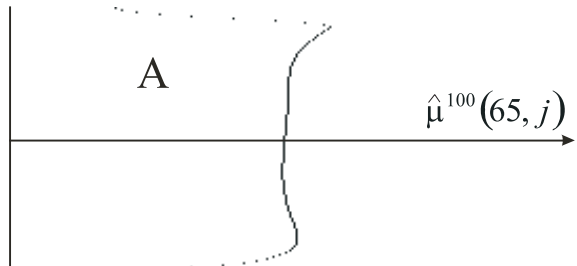

(a)

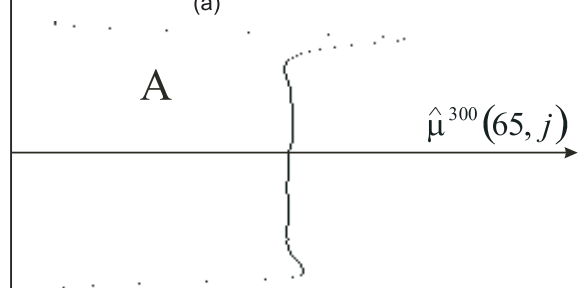

(b)

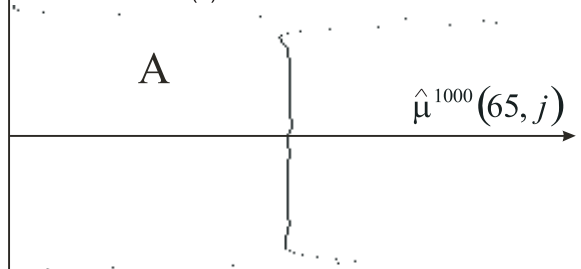

(c)

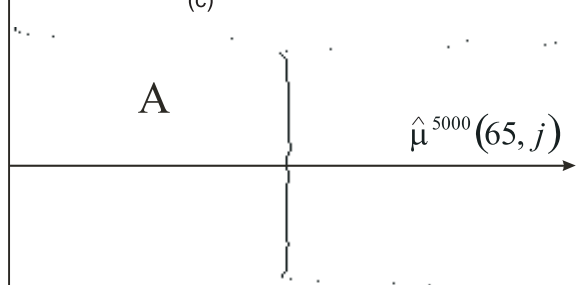

(d)

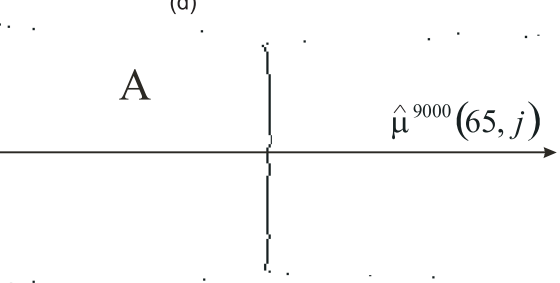

(e)

Fig. 7. View of the reconstructed image after: (a) $t=100$ iterations, (b) $t=300$ iterations, (c) $t=1000$ iterations, (d) $t=5000$ iterations, (e) $t=9000$ iterations (window: $C=1.0, W=0.2$ ).

Table 1. Results of reconstruction algorithms evaluated by the error measures (28) and (29).

\begin{tabular}{|l|c|c|c|c|}
\hline \multirow{2}{*}{ Algorithm } & \multicolumn{2}{|c|}{ with noise } & \multicolumn{2}{c|}{ without noise } \\
\cline { 2 - 5 } & MSE & SNR & MSE & SNR \\
\hline \hline Neural network algorithm (30,000 iterations) & 0.01100 & 17.62480 & 0.01072 & 17.70703 \\
\hline $\begin{array}{c}\text { Convolution back-projection algorithm } \\
\text { (Shepp-Logan convolution kernel) }\end{array}$ & 0.01237 & 17.11509 & 0.01219 & 17.14408 \\
\hline
\end{tabular}




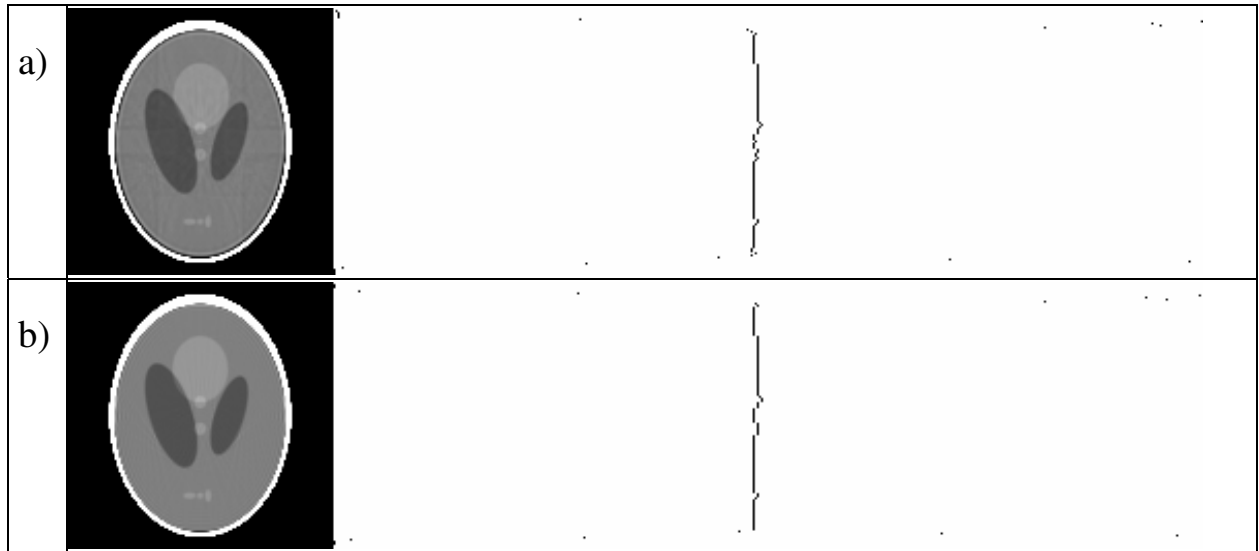

Fig. 9. View of the reconstructed image from the same projections using: (a) neural network structure, $w=6.2 \times 10^{10}$, (b) convolution back-projection algorithm.

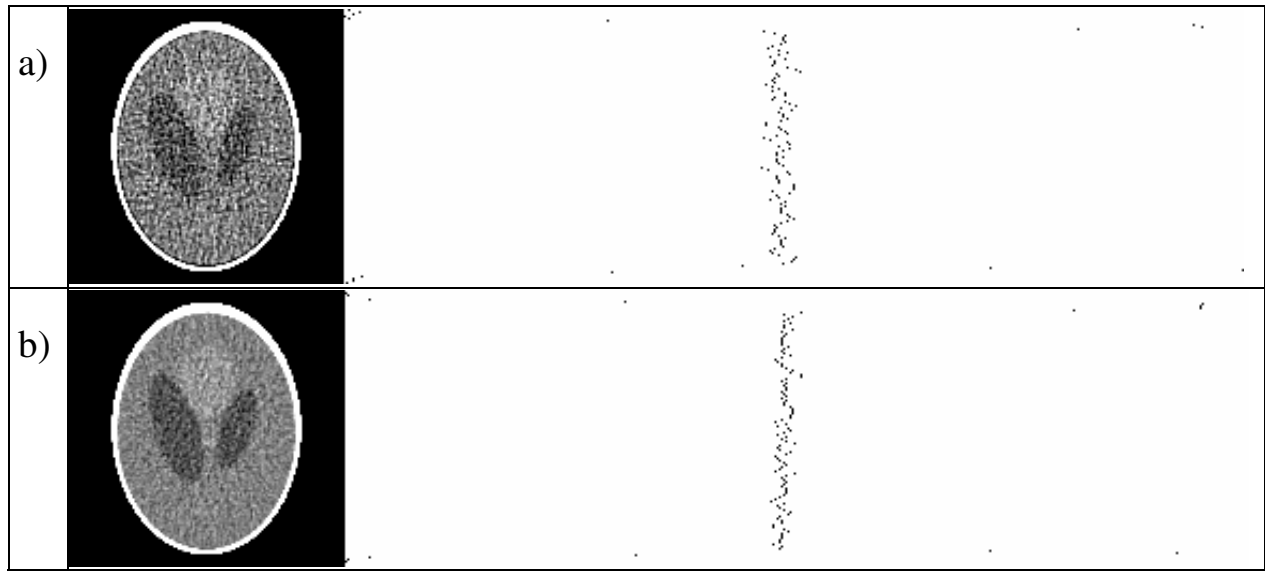

Fig. 10. View of the reconstructed image from the same projections using: (a) neural network structure, $w=6.2 \times 10^{10}$, (b) convolution back-projection algorithm.

on Artificial Intelligence and Soft Computing, Zakopane, Poland, LNAI, Springer, Berlin, pp. 890-898.

Cormack A.M. (1963). Representation of a function by its line integrals with some radiological application, Journal of Applied Physics 34: 2722-2727.

Gordon R., Bender R. and Herman G.T. (1970). Algebraic reconstruction techniques (ART) for three-dimensional electron microscopy and X-ray photography, Journal of Theoretical Biology 29: 471-481.

Hopfield J.J. (1982). Neural networks and physical systems with emergent collective computational abilities, Proceedings of the National Academy of Sciences of the USA 79: 2554-2558.

Ingman D. and Merlis Y. (1992). Maximum entropy signal reconstruction with neural networks, IEEE Transactions on Neural Networks 3: 195-201.

Jaene B. (1991). Digital Image Processing - Concepts, Algoritms and Scientific Applications, Springer, Berlin, Heidelberg.
Jain A.K. (1989). Fundamentals of Digital Image Processing, Prentice Hall, Englewood Cliffs, NJ.

Kaczmarz S. (1937). Angeneaherte Aufloesung von Systemen Linearer Gleichungen, Bulletin de l'Académie Polonaise des Sciences et Lettres 35: 355-357.

Kak A.C. and Slanley M. (1988). Principles of Computerized Tomographic Imaging, IEEE Press, New York.

Kerr J.P. and Barlett E.B. (1995a). A statistically tailored neural network approach to tomographic image reconstruction, Medical Physics 22: 601-610.

Kerr J.P. and Barlett E.B. (1995b). Medical image processing utilizing neural networks trained on a massively parallel computer, Computers in Biology and Medicine 25: 393-403.

Kerr J.P. and Barlett E.B. (1995c). Neural network reconstruction of single-photon emission computed tomography images, Journal of Digital Imaging 8: 116-126.

Kingston A. and Svalbe I. (2003). Mapping between digital and continuous projections via the discrete Radon transform in Fourier space, Proceedings of the 7-th Conference on Dig- 
ital Image Computing: Techniques and Applications, Sydney, pp. 263-272.

Knoll P., Mirzaei S., Muller A., Leitha T., Koriska K., Kohn H. and Neumann M. (1999). An artificial neural net and error backpropagation to reconstruct single photon emission computerized tomography data, Medical Physics $\mathbf{2 6}$ : 244-248.

Lewitt R.M. (1983). Reconstruction algorithms: Transform methods, Proceeding of the IEEE 71(3): 390-408.

Luo F.-L. and Unbehauen R. (1998). Applied Neural Networks for Signal Processing, Cambridge University Press, Cambridge, UK

Munllay M.T., Floyd C.E., Bowsher J.E. and Coleman R. E. (1994). An artificial neural network approach to quantitative single photon emission computed tomographic reconstruction with collimator, attenuation and scatter compensation, Medical Physics 21: 1889-1899.
Ramachandran G.N. and Lakshminarayanan A.V. (1971). Threedimensional reconstruction from radiographs and electron micrographs: II. Application of convolutions instead of Fourier transforms, Proceedings of the National Academy of Sciences of the USA 68: 2236-2240.

Srinivasan V., Han Y.K. and Ong S.H. (1993). Image reconstruction by a Hopfield neural network, Image and Vision Computing 11(5): 278-282.

Wang Y. and Wahl F.M. (1997). Vector-entropy optimizationbased neural-network approach to image reconstruction from projections, IEEE Transaction on Neural Networks 8(5): 1008-1014.

Received: 25 June 2007

Revised: 30 July 2007 
